\title{
Oleuropein alleviates gestational diabetes mellitus by activating AMPK signaling
}

\author{
Zhiwei Zhang*, Hui Zhao* and Aixia Wang \\ Department of Obstetrics and Gynecology, Liaocheng People's Hospital, Liaocheng, Shandong, China \\ Correspondence should be addressed to A Wang: wangaixia12@163.com \\ *(Z Zhang and $\mathrm{H}$ Zhao contributed equally to this work)
}

\begin{abstract}
Background: Gestational diabetes mellitus (GDM) has a high incidence rate among pregnant women. The objective of the study was to assess the effect of plant-derived oleuropein in attenuating inflammatory and oxidative stress of GDM.

Methods: Oleuropein was administered to GDM mice at the doses of 5 or $10 \mathrm{mg} / \mathrm{kg} /$ day. Body weight, blood glucose, insulin and hepatic glycogen levels were recorded. To evaluate the effect of oleuropein in reducing oxidative stress, ELISA was used to measure the hepatic oxidative stress markers. The inflammation levels of GDM mice were evaluated by measuring serum levels of IL- 6 and TNF- $\alpha$ by ELISA and mRNA levels of IL- $1 \beta$, TNF- $\alpha$ and IL- 6 by real-time PCR (RT-PCR). The AMP-activated protein kinase (AMPK) signaling pathway was assessed by Western blot. Gestational outcome was analyzed through comparing litter size and birth weight.

Results: Oleuropein attenuated the elevated body weight of GDM mice and efficiently reduced blood glucose, insulin and hepatic glycogen levels. Oxidative stress and inflammation were alleviated by oleuropein treatment. The AMPK signaling was activated by oleuropein in GDM mice. Gestational outcome was markedly improved by oleuropein treatment.

Conclusions: Our study suggests that oleuropein is effective in alleviating symptoms of GDM and improving gestational outcome in the mouse model. This effect is achieved by attenuating oxidative stress and inflammation, which is mediated by the activation of the AMPK signaling pathway.
\end{abstract}

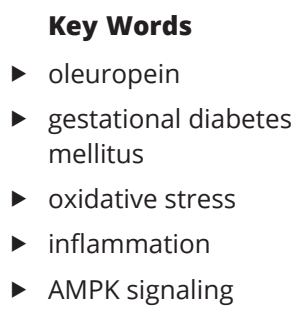

Endocrine Connections (2021) 10, 45-53

\section{Introduction}

Gestational diabetes mellitus (GDM) manifests as various degrees of hyperglycemia during pregnancy. GDM has a high incidence rate and is by far one of the leading causes of neonatal and maternal mortality (1). The etiology of GDM has not been fully elucidated. Previous studies have suggested that antagonism of insulin by hormones secreted by the placenta, such as placental prolactin (HPL), estrogen, progesterone, etc., mainly contributes to pathogenesis of GDM (2). In treating diabetes, insulin is primarily used to lower blood glucose (3), but the dosage of insulin widely varies among individuals, which in addition to insulin resistance compromises its clinical utility in reducing maternal and neonatal complications (4). There is a continuous search for new biocompatible drugs, with the capabilities of lowering blood glucose and countering insulin resistance, for the clinical management of GDM.

Plant-derived pharmaceutics are one of the major sources of new drugs for various human diseases including $\operatorname{GDM}(5,6)$. Numerous studies have indicated that the incidence rates of certain cancers and coronary heart diseases are the lowest in the Mediterranean basin
This work is licensed under a Creative Commons Attribution-NonCommercial 4.0 International License. ded from Bioscientifica.com at 04/26/2023 11:21:37AM 
$(7,8,9)$. Mediterranean coastal diet is rich in olive products, and oleuropein, the main phenolic component of Olea europaea L., has been widely used in clinics for its anti-inflammatory, anti-oxidative (10), heart and nerve protective $(11,12)$, and anti-cancer effects (13). Inflammation and oxidative stress are two common targets for therapies against GDM. Placental inflammation and GDM have been closely correlated, and both of them play essential roles in shaping the fetal environment (14). Inflammation is highly regulated in normal pregnancy, which is of critical importance in maintaining a hospitable environment for fetal development. GDM has been linked to dysregulated expression and activity of placental nutrient transport proteins, which may stem from altered inflammatory status of the placenta, mother and fetus, resulting in comorbidities observed in these pregnancies. Previous studies have demonstrated the efficacy of oleuropein in different animal models of diabetes, such as the alloxan-induced (15) and obesityinduced (16) diabetic rat models, making oleuropein a potential drug for GDM clinical management.

The suppression of AMP-activated protein kinase (AMPK) signaling in the liver is one of the characteristics of GDM. AMPK is one of the enzymes that regulate the cellular response to metabolic stress, and inhibited AMPK signaling has been found to correlate with insulin resistance, inflammation $(17,18)$ and oxidative stress (19). For example, activating AMPK has been found to attenuate LPS-induced release of pro-inflammatory cytokines (18). AMPK activation is also demonstrated to be a cellular reprogramming process in response to mitochondrial dysfunction to alleviate oxidative stress (19). Therefore, AMPK has been considered a promising target to regulate diseases characterized by elevated inflammation and oxidative stress. Previous studies have also implicated the link between AMPK suppression and reduced glucose uptake (20), which is a contributing factor to hyperglycemia. A number of studies have employed AMPK as a target to treat $\operatorname{GDM}(21,22,23)$. Given the important role of AMPK in GDM and the potential of oleuropein in re-activating AMPK signaling, as suggested by a previous study (24), a comprehensive evaluation of the effects of oleuropein in alleviating symptoms of GDM is warranted.

In this study, we aimed to study the efficacy of oleuropein in treating GDM in a genetically modified mouse model of GDM. GDM symptoms, including increased blood glucose levels, body weight, plasma insulin levels and hepatic glycogen contents, were compared in GDM mice with or without oleuropein treatment. Oxidative stress was examined by measuring liver oxidative stress markers. Inflammatory levels were evaluated by assessing interleukin-1 $\beta$ (IL-1 $\beta$ ), IL-6 and tumor necrotic factor $\alpha$ (TNF- $\alpha$ ) levels in the serum. As a mechanistic study, we also examined the link between the AMPK signaling pathway and oleuropein treatment. The results of the study could provide insights into the efficacy of oleuropein in alleviating GDM and paving the path for its clinical translation.

\section{Materials and methods}

\section{Animals}

C57BL/KsJ Lep ${ }^{\mathrm{db} /+}(\mathrm{db} /+)$ and C57BL/KsJ+/+ (WT) mice of 6-8 weeks old were purchased from Jackson Laboratories. The mice were fed with a high-fat diet (17\% fat, $47 \%$ carbohydrate and $29 \%$ protein, Harlan Teklad). The animals were housed in an air-conditioned room with controlled temperature of $22 \pm 2^{\circ} \mathrm{C}$ and maintained on a $12 \mathrm{~h}$ light:12 $\mathrm{h}$ darkness cycle with free access to food and water. Female mice of 10-12 weeks old were mated in individual cages. The day following mating was denoted as gestation day (GD) 0 . Gestational mice were randomly grouped as the following ( $n=15$ per group): normal gestation control (WT mice), GDM group C57BL/KsJ $\mathrm{Lep}^{\mathrm{db} /+}(\mathrm{db} /+)$, GDM+low-dose oleuropein group (5 mg/ $\mathrm{kg} /$ day, i.p. oleuropein: Sigma-Aldrich) and GDM+highdose oleuropein group $(10 \mathrm{mg} / \mathrm{kg} /$ day, i.p.). Saline was administered i.p. in the normal pregnancy control group and the GDM group. The mice were subjected to measurement of intraperitoneal glucose tolerance test (IPGTT) and intraperitoneal insulin tolerance test (IPITT) on GD10, detection of the levels of inflammatory factors on GD15, and assessment of the serum glucose content on GD20. A 5 days gap was included between each measurement to ensure sufficient recovery of the mice and to exclude interference from the invasive measurements. The study was approved by the Ethics Committee of Liaocheng People's Hospital (LCRMYY-027a). Oleuropein (Sigma-Aldrich Chemical, 98\%) was dissolved in dimethyl sulfoxide, then diluted with PBS. The final concentration was $5 \mathrm{mg} / \mathrm{mL}$.

\section{Intraperitoneal glucose and insulin tolerance test}

IPGTT and IPITT were conducted on GD10. For IPGTT, glucose was i.p. administrated at the dose of $2.0 \mathrm{~g} / \mathrm{kg}$ after $6 \mathrm{~h}$ of fasting. An ACCU-CHEK dominant glucose meter

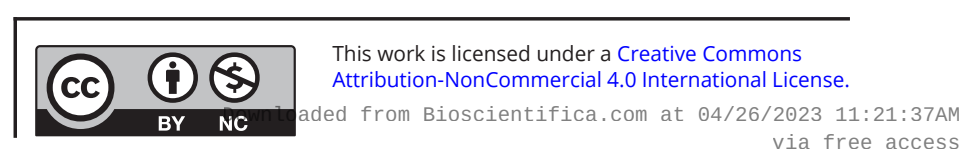


(Roche Diagnostics) was used to measure glucose levels at baseline and after glucose injection (at 30, 60, 90 and 120 min after injection). For IPITT, insulin was administered i.p. at the dose of $0.75 \mathrm{U} / \mathrm{kg}$ at $6 \mathrm{~h}$ after fasting and blood glucose concentrations were measured at baseline and after insulin injection (at 30, 60, 90 and 120 min after injection).

\section{Lipid biochemistry}

Lipids were extracted from blood in a $0.125 \mathrm{M}$ potassium phosphate buffer and normalized to total protein content. Cholesterol, triglycerides, high-density lipoprotein (HDL) cholesterol and low-density lipoprotein (LDL) cholesterol were measured in the plasma using a Unicel DxC 800 autoanalyzer (Beckman-Coulter, the Netherlands) and dedicated kits.

\section{Hepatic glycogen content measurement}

The liver tissue was processed using a commercial kit (BioVision). A microplate reader (Spectra Max 384, Molecular Devices, USA) was used to measure the absorbance of hepatic glycogen at $340 \mathrm{~nm}$ of wavelength after liver lysates were mixed with the analytical reagent using the manufacturer's protocol.

\section{Serum glucose and insulin measurements}

At GD20, glucose levels were analyzed using a glucose meter (Roche Diagnostics). An ultra-sensitive mouse insulin ELISA kit (ALP CO Diagnostics) was used to measure insulin levels.

\section{Pathological investigation}

First, animals were anesthetized by ether, then the pancreatic islets were extracted. Pancreatic islets were fixed in $10 \%$ buffered formalin, embedded in paraffin, sectioned $(4 \mu \mathrm{m})$ and stained with hematoxylin and eosin (H\&E).

\section{ELISA}

At GD15, the ELISA kits acquired from R\&D Systems were used to quantify serum levels of TNF- $\alpha$, IL- $1 \beta$ and IL- 6 according to the manufacturer's protocols.

\section{Real-time quantitative polymerase chain reaction}

Trizol reagent (Thermo Fisher) was used to extract total RNA from skeletal muscles according to the manufacturer's protocol, followed by RT of the RNA using the SuperScript ${ }^{\circledR}$ III system (Thermo Fisher). Real-time PCR was conducted on the QuantStudio 5 Thermocycler (Thermo Fisher) using the QuanttiTect SYBR Green Kit (Qiagen). The following primers were used in this study: IL-1 $\beta$, f: 5'-AACCTGCTGGTGTGTGACACTCTC-3', r: 5'-CAGC ACGAGGCTTTTTTGTTGT-3'; IL-6, f: 5'-ACAACCACGG CCCCTTCCCTACTT-3', r: 5'-CACGATTTCCCAGAACAT GTG-3'; TNF- $\alpha$, f: 5'-GCCTCTTCTCATTCCTGCTTG-3', r: 5'-CTGATGAGAGGGAGGCCATT-3'; $\beta$-actin f: 5'-CGTG CGTGACATCAAAGAGAA-3', r: 5'-TGGATGCCACAGGA TTCCAT-3'. The amplification protocol was as follows: pre-incubation at $98^{\circ} \mathrm{C}$ for $1 \mathrm{~min}$, followed by 40 cycles at $98^{\circ} \mathrm{C}$ for $10 \mathrm{~s}, 56^{\circ} \mathrm{C}$ for $20 \mathrm{~s}$, and $72^{\circ} \mathrm{C}$ for $30 \mathrm{~s}$. The relative mRNA expressions of targeted genes were calculated using the comparative $2^{-\Delta \Delta C t}$ method. $\beta$-Actin was used as the internal control. The mRNA expression was first normalized using $\beta$-actin mRNA values, and then the mRNA expression of individual genes was normalized to that of the WT mice.

\section{Oxidative stress assessment}

Levels of superoxide dismutase (SOD), thiobarbituric acid reactive substances (TBARS), catalase (CAT) and glutathione (GSH) in liver lysates were determined using commercially available kits (Nanjing Jiancheng, China) according to manufacturer's protocol.

\section{Western blot}

Protein samples were harvested from liver tissues. After blocking with 5\% non-fat milk, the PVDF membranes containing resolved proteins were incubated overnight at $4^{\circ} \mathrm{C}$ using rabbit anti-mouse antibodies against AMPK (1:1000, Abcam), phosphorylated HDAC4 (1:1000, Abcam), p-AMPK (1:1000, Santa Cruz), HDAC4 (1:1000, Santa Cruz), G6Pase (1:1000, Abcam) and $\alpha$-microtubule protein (1:1000, Santa Cruz). The membranes were then incubated with peroxidase-conjugated secondary antibodies (Sigma-Aldrich) for $1 \mathrm{~h}$ and developed using an ECL system acquired from GE Healthcare Life Science. Relative expression was calculated using NIH-Image J1.51p 22 (NIH).

\section{Statistical analysis}

Data were presented as mean \pm s.D. The Prism Graphpad software was used for statistical analysis. Analysis of differences between two groups was performed using

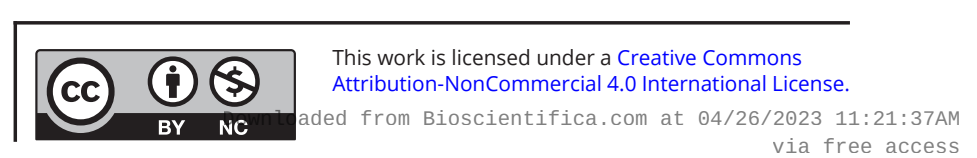


ANOVA (one or two-way) with a post hoc test, and statistically significant comparisons were denoted as the following: ${ }^{\star} P<0.05,{ }^{* \star} P<0.01$.

\section{Results}

\section{Oleuropein attenuates weight gain in GDM mice}

The experimental design of the study is shown in Supplementary Fig. 1 (see section on supplementary materials given at the end of this article). Mice were treated with two doses of oleuropein (low dose: 5 $\mathrm{mg} / \mathrm{kg}$; high dose: $10 \mathrm{mg} / \mathrm{kg}$ ) based on a pilot study investigating the effects of oleuropein of $5-15 \mathrm{mg} / \mathrm{kg}$ on lowering fasting blood glucose and blood insulin levels (Supplementary Fig. 2). We examined the body weight of four groups of mice at GD0, GD10 and GD20, and the body weight gain on GD20 was compared to GD0. We found that GDM mice showed significantly higher gain in body weight compared to normal WT mice as a result of GDM, but this gain was significantly reduced by oleuropein treatment $(P<0.01$, compared to GDM group). This reduction was further augmented by higher oleuropein concentrations $(P<0.01$, compared to GDM group, Fig. 1A and B). Photographs of mice in different groups (Fig. 1C) also showed that oleuropein alleviated obesity in GDM mice.

\section{Oleuropein attenuates serum glucose and insulin intolerance}

As shown in Fig. 2A, the GDM group exhibited higher blood glucose level than the normal pregnancy control group, and the high-dose oleuropein treatment group had significantly reduced blood glucose compared to the GDM group ( $P<0.01$ compared to GDM group), and low-dose oleuropein treatment also induced certain reduction in the blood glucose level. As shown in Fig. 2B, by measuring the blood glucose levels at various time points after insulin injection, we found that the high-dose oleuropein treatment group had significant reduction in blood glucose in response to insulin compared to the GDM group $(P<0.01$ compared to GDM group). All these data suggested that oleuropein improved glucose and insulin intolerance in GDM mice. On GD20, fasting glucose and insulin levels in the GDM group were significantly higher than in the healthy gestation control group (Fig. 2C and D). The increased glucose and insulin levels under fasting in GDM mice could be significantly

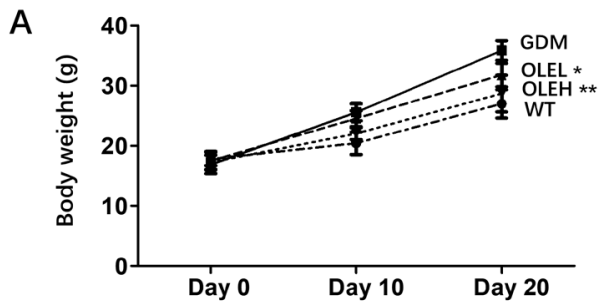

B

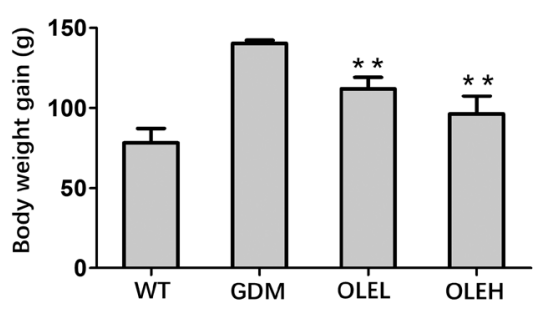

C

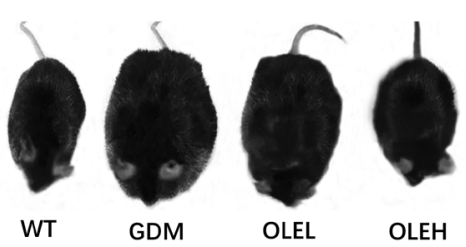

Figure 1

Oleuropein administration alleviates body weight in GDM mice. (A) Body weight of pregnant mice was recorded on GD0, GD10, and GD20 in the WT, GDM + Vehicle group (GDM), GDM + 5 mg/kg/day Oleuropein group (OLEL) and GDM + $10 \mathrm{mg} / \mathrm{kg} /$ day Oleuropein group (OLEH). (B) Comparison of body weight gains from GD0 to GD20. (C) Representative photos before labor. $n=10$ for each group. Data were presented as mean \pm s.D. ${ }^{*} P<0.05,{ }^{*} * P<0.01$ vs GDM group.

attenuated by both the low-dose $(P<0.05)$ and highdose oleuropein treatments $(P<0.01)$ (Fig. 2C and D). While the GDM group had a significant reduction in the hepatic glycogen contents compared to the WT group, oleuropein exerted a dose-dependent effect in restoring the hepatic glycogen levels (Fig. 2E, $P<0.05$ for low-dose oleuropein and $P<0.01$ for high-dose oleuropein). Next, we compared the efficacy of oleuropein to metformin, which is a commonly used drug for managing insulin resistance. Our study showed that oleuropein exhibited comparable efficacy to metformin in alleviating GDM symptoms (Supplementary Fig. 3A, B, C, D, E and F). Further, H\&E staining of the pancreatic islets indicated that normal islets were evenly distributed, displaying intact structures and regular edges. In contrast, islets in the GDM group were characterized by swelling, hyperplasia, irregular and blurry edges. Treatment with $10 \mathrm{mg} / \mathrm{kg}$ oleuropein was able to reduce pathological changes in these islets (Fig. 2F).

We also assessed the influence of oleuropein on weight gain/food intake of the mice and showed that GDM mice exhibited a higher ratio of weight gain/food
This work is licensed under a Creative Commons Attribution-NonCommercial 4.0 International License. ded from Bioscientifica.com at 04/26/2023 11:21:37AM via free access 


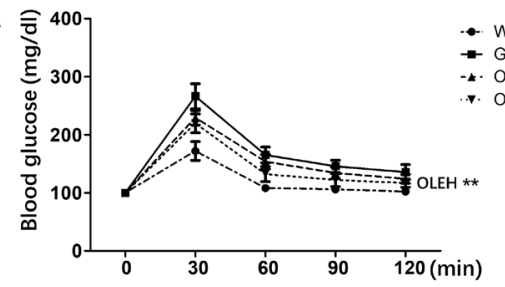

C

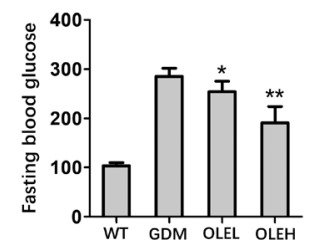

$\mathrm{F}$
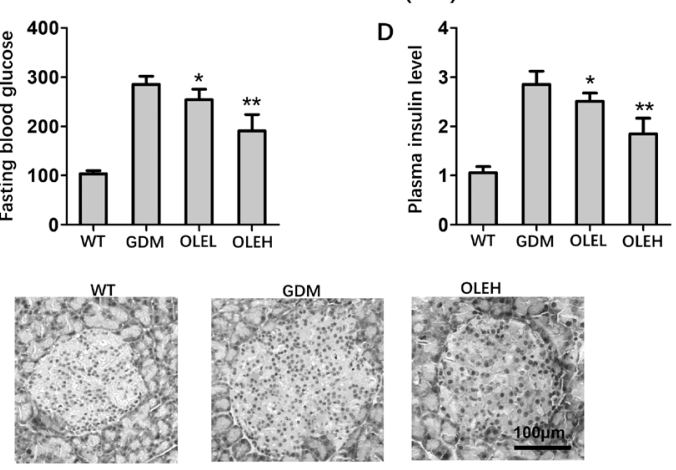

OLEH

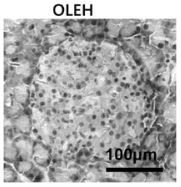

intake, which is a common symptom of insulin resistance. Both low-dose and high-dose of oleuropein were able to significantly decrease the weight gain/food intake ratios (Supplementary Fig. 4).

\section{Oleuropein reduces serum lipid levels and attenuates oxidative stress in GDM mice}

Maternal plasma concentrations of total cholesterol (TC) (Fig. 3A), triglyceride (TG) (Fig. 3B), HDL cholesterol (Fig. 3C) and LDL cholesterol (Fig. 3D) were measured on GD20. Our result indicated that GDM mice were characterized by upregulation of all the four lipids and oleuropein was efficient at reducing serum lipid levels, particularly at a high dose (10 $\mathrm{mg} / \mathrm{kg})$.

Liver TBARS levels were significantly elevated in the GDM group compared to the normal pregnancy group. Treatment with high-dose oleuropein attenuated the increased liver TBARS (Fig. 4A). In contrast, GSH, SOD and CAT, which were lower in GDM mice, were restored by oleuropein treatment (Fig. 4, B, C and D).

\section{Oleuropein inhibits inflammatory response in GDM mice}

We further examined the effects of oleuropein in attenuating inflammatory responses in GDM mice. In our study, low-dose and high-dose oleuropein significantly reduced the expression levels of IL-6, IL-1 $\beta$ and TNF- $\alpha$ in the serum (Fig. 5A, B and C) and skeletal muscles (Fig. 5D, E and F) of GDM mice, and this effect was more pronounced in the high-dose group.

\section{Oleuropein promotes AMPK activation in the liver}

To investigate the mechanism underlying the action of oleuropein, livers were taken on GD20 to examine the effects of oleuropein on the AMPK signaling pathway (Fig. 6A, B, C and D). AMPK activation was found to be suppressed in the GDM group compared to the WT group, while oleuropein significantly restored the expression of p-AMPK. In the GDM mice, there were higher levels of HDAC4 activation and G6Pase upregulation, whereas oleuropein significantly reduced the upregulation in these proteins in a dose-dependent manner. These data suggested
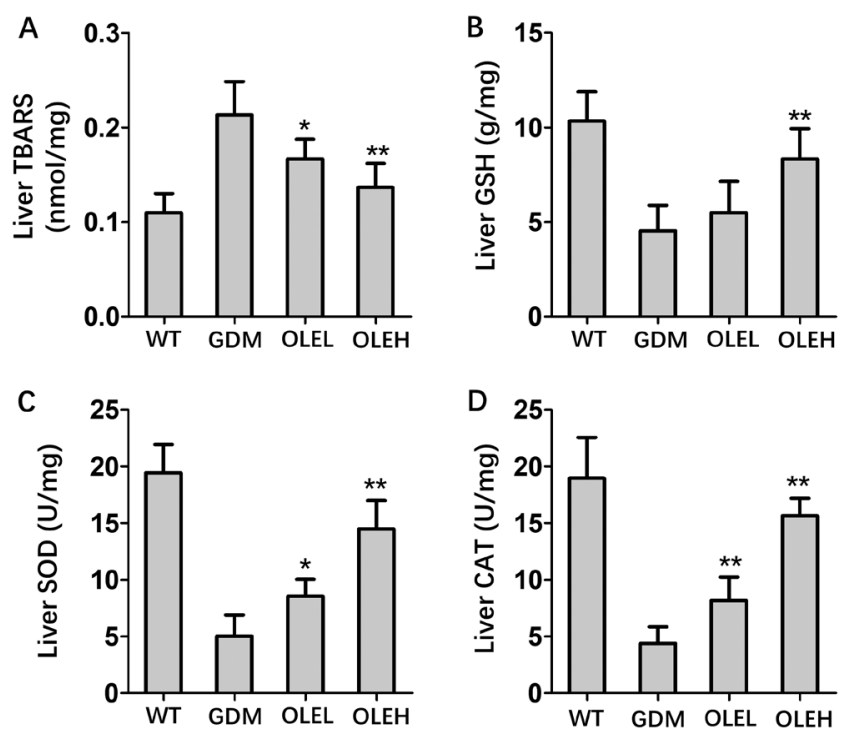

\section{Figure 4}

Oleuropein alleviates oxidative stress on GDM mice. TBARS (A), GSH (B), SOD (C) and CAT (D) activities were assayed. $n=10$ for each group. Data were presented as mean \pm S.D. ${ }^{*} P<0.05$, ${ }^{*} P<0.01$ vs GDM group.

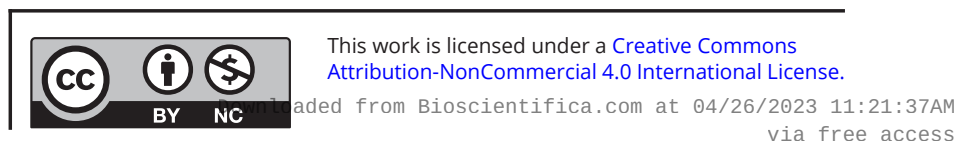


that the ameliorative effects of oleuropein on GDM mice could be mediated by the activation of the AMPK signaling.

\section{Effects of oleuropein on reproductive function in GDM mice}

To examine the effects of oleuropein on gestational outcome, we evaluated the litter size and birth weight of GDM mice with or without oleuropein treatment. Oleuropein significantly restored the decreased litter size (Fig. 7A) and increased birth weight (Fig. 7B) as a result of GDM, suggesting that the anti-inflammatory and antioxidative effects of oleuropein translated to improved gestational outcome in GDM mice.

\section{Discussion}

Maternal hormonal and metabolic changes associated with GDM profoundly alter the intrauterine environment, resulting in abnormal fetal growth, serious metabolic consequences, increased risk of abnormal glucose tolerance and obesity during adolescence and later in life. Inspired by the well-known health benefit of the Mediterranean diet, we hereby assessed the potency of oleuropein, a major phenolic extract of olives that has been shown to be safe to pregnant mice (25), in treating GDM in a genetic modified Lep ${ }^{\mathrm{db} /+}(\mathrm{db} /+)$ mouse model. This model harbors a mutated leptin receptor gene Lepr leading to abnormalities closely resembling human GDM symptoms during pregnancy and has been extensively used to evaluate drugs for treating GDM (26). Besides hyperglycemia, obesity and insulin resistance, fetal development was also impaired in this genetic mouse model. We showed that oleuropein effectively attenuated increased body weight of pregnant mice, meanwhile lowering blood glucose level, insulin resistance and increasing hepatic glycogen contents. This finding is in agreement to previous reports on the effects of oleuropein in reducing blood glucose of other GDM models, such as alloxan-induced and obesity-induced diabetic rats (15).

Inflammation and oxidative stress are two major factors that contribute to the pathogenesis of GDM, and we showed that oleuropein treatment was capable of restoring the elevated inflammatory response and oxidative stress. Dysregulation of inflammatory response and oxidative stress during pregnancy may be a result of altered hormone levels and energy metabolism in pregnant women (27). Increased hormone production can promote lipolysis, which potentially leads to abnormalities in the secretion of inflammatory factors, lipid metabolism, glucose metabolism and insulin resistance (28). Dysregulated protein and lipid oxidation could also contribute to excessive oxidative stress, which further disrupts hemostasis (29). TNF- $\alpha$ is one of the earliest cytokines in the inflammatory response, and it acts as the initiating factor and activates the secretion of IL-6, an important pro-inflammatory factor in inflammatory response (30). Patients with GDM have significantly higher levels of inflammatory factors, such as IL-6 and TNF- $\alpha$, than those with normal pregnancy and are in a chronic inflammatory state. Herein, we showed that the oxidative stress in the liver, measured by liver TBARS, GSH, SOD and CAT levels, were attenuated by oleuropein treatment. Meanwhile, IL-6, IL-1 $\beta$ and TNF- $\alpha$ levels in the serum and skeletal muscles were also reduced. We included measurement of inflammatory factors in both the serum and skeletal muscles, as oleuropein has been reported to
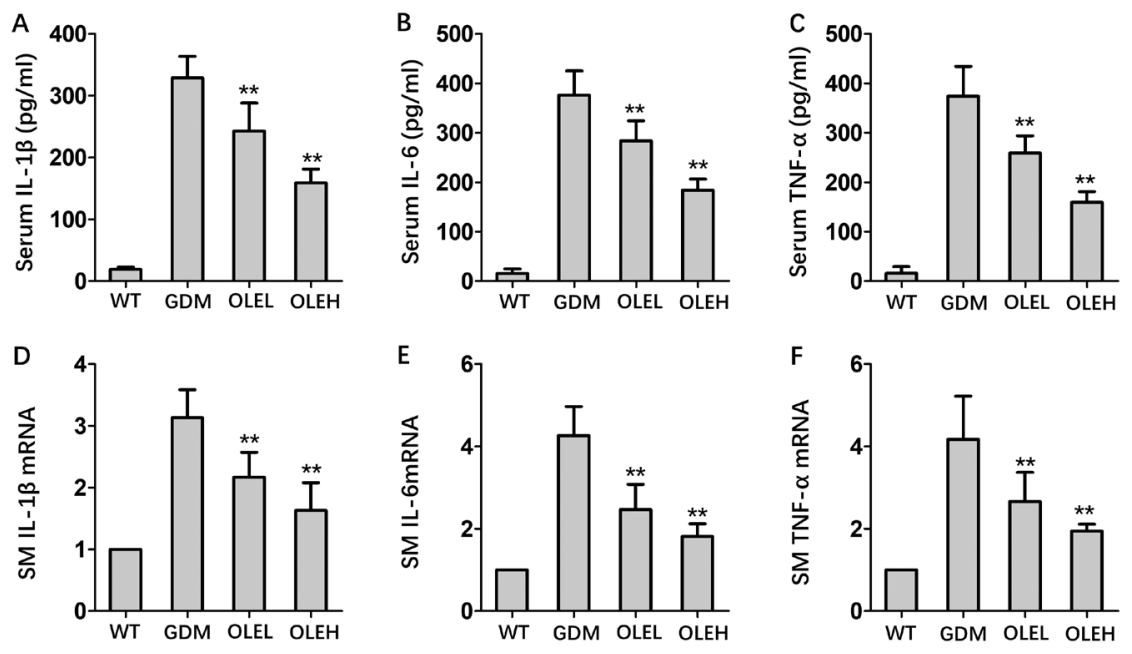

Figure 5

Oleuropein inhibits GDM-associated inflammation. Serum concentration of IL-1 $\beta(A)$, IL-6 (B), and TNF- $\alpha$ (C) in different groups. Relative mRNA levels of IL-1 $\beta(D)$, IL-6 (E), and TNF- $\alpha$ (F) were detected by RT-PCR in skeletal muscle (SM). $n=5$ for each group. Data were presented as mean \pm S.D. ${ }^{*} P<0.05, * \star P<0.01$ vs GDM group. 


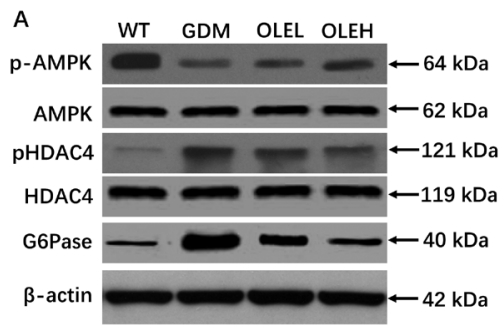

C

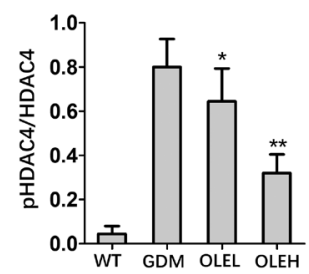

B

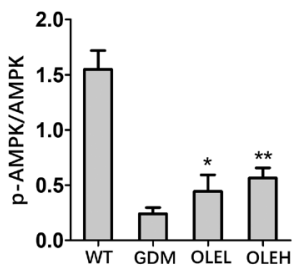

D

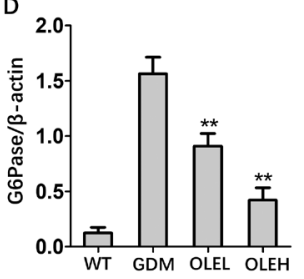

Figure 6

Effects of oleuropein on AMPK signaling pathway in GDM mice. (A) Representative Western blot images for analyzing the expression of total p-AMPK, AMPK, total HDAC4, pHDAC4, and G6Pase on GD20. (B, C and D) Quantitative analysis after being normalized by $\beta$-actin. $n=5$ for each group. Data were presented as mean \pm S.D. ${ }^{*} P<0.05, * \star P<0.01$ vs GDM group.

decrease inflammation in skeletal muscles as well (31). Together, these data have corroborated the potency of oleuropein as an anti-oxidative and anti-inflammatory drug suggested by previous studies and shed light on the utility of oleuropein in maintaining tissue hemostasis.

To explore the molecular mechanism of oleuropein in the treatment of GDM, we focused on the AMPK signaling pathway. A number of studies have employed AMPK as a target to treat $\operatorname{GDM}(21,22,23)$. Our data are consistent with previous findings demonstrating that GDM is characterized by suppressed AMPK signaling in the liver, and oleuropein was capable of re-activating AMPK signaling (24). Previous studies have established two possible mechanisms responsible for the hypoglycemic effect of oleuropein: (1) ameliorated glucose-induced insulin release, and (2) enhanced peripheral glucose uptake (32). Oleuropein has been observed to accelerate cellular uptake of glucose, leading to decreased plasma glucose. The link between the AMPK signaling and glucose uptake has also been established (20), which explains the enhanced glucose uptake after oleuropein treatment. Further, inhibited AMPK signaling is also associated with insulin resistance, inflammation and oxidative stress. Therefore, these data support the attenuated symptoms of GDM by oleuropein. Indeed, the reduced glucose levels, inflammation and oxidative stress led to improved gestational outcome, as shown by the increased litter size and decreased birth weight of GDM mice. It should be noted that other mechanisms of

oleuropein in ameliorating diabetes have been proposed. For example, oleuropein was shown to alleviate insulin resistance by promoting the translocation of glucose transporter 4 (31). It should also be noted that oleuropein has been found to activate other signaling pathways, such as the AKT signaling (33) and the $\beta$-adrenergic signaling (34), but whether these signaling pathways are also involved in the regulation of GDM has not been investigated in our study. In addition, cellular-level study on the effects of oleuropein on islets, potentially using human islet amyloid as reported recently (34), is needed to corroborate the findings of the study.

\section{Conclusions}

To conclude, here we report the use of a plant extract oleuropein for the treatment of GDM, where oleuropein is shown to alleviate symptoms of GDM, restore normal glucose level and insulin sensitivity, while decreasing inflammatory responses and oxidative stress. Gestational outcome is also improved by oleuropein treatment. Oleuropein may exert its therapeutic effects through the activation of the AMPK signaling pathway.
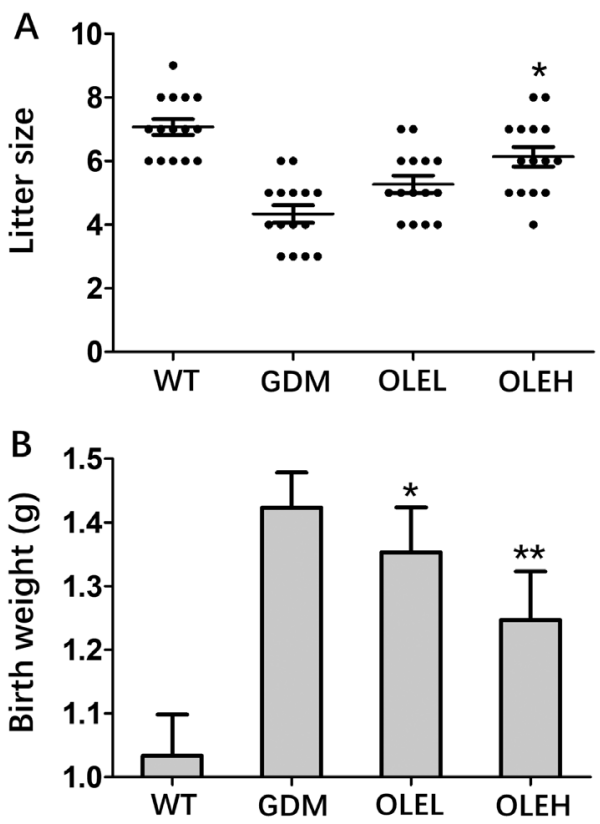

Figure 7

Oleuropein improves reproductive outcome in GDM mice. Litter size (A) and body weight after birth (B) were recorded in different groups $n=15$ for each group. Data were presented as mean \pm S.D. ${ }^{*} P<0.05, * \star P<0.01$ vs GDM group. 


\section{Supplementary materials}

This is linked to the online version of the paper at https://doi.org/10.1530/ EC-20-0466.

\section{Declaration of interest}

The authors declare that there is no conflict of interest that could be perceived as prejudicing the impartiality of the research reported.

\section{Funding}

This research did not receive any specific grant from any funding agency in the public, commercial or not-for-profit sector.

\section{References}

1 American Diabetes Association. Gestational diabetes mellitus. Diabetes Care 200326 (Supplement 1) S103-S105. (https://doi. org/10.2337/diacare.26.2007.s103)

2 Tawfeek MA, Alfadhli EM, Alayoubi AM, El-Beshbishy HA \& Habib FA. Sex hormone binding globulin as a valuable biochemical marker in predicting gestational diabetes mellitus. BMC Women's Health 201717 18. (https://doi.org/10.1186/s12905-017-0373-3)

3 Brown J, Grzeskowiak L, Williamson K, Downie MR \& Crowther CA. Insulin for the treatment of women with gestational diabetes. Cochrane Database of Systematic Reviews 201711 CD012037. (https:// doi.org/10.1002/14651858.CD012037.pub2)

4 Tumurbaatar B, Poole AT, Olson G, Makhlouf M, Sallam HS, Thukuntla S, Kankanala S, Ekhaese O, Gomez G, Chandalia M, et al. Adipose tissue insulin resistance in gestational diabetes. Metabolic Syndrome and Related Disorders 201715 86-92. (https://doi. org/10.1089/met.2016.0124)

5 Raji M \& Chen Z. Effects of abiotic elicitors on the production of bioactive flavonols in Emilia sonchifolia. STEMedicine 1 e33. (https:// doi.org/10.37175/stemedicine.v1i2.33)

6 Sajadimajd S, Bahrami G, Daglia M, Nabavi SM, Naseri R \& Farzaei MH. Plant-derived supplementary carbohydrates, polysaccharides and oligosaccharides in management of diabetes mellitus: a comprehensive review. Food Reviews International 201935 563-586. (https://doi.org/10.1080/87559129.2019.1584818)

7 Izadi V, Tehrani H, Haghighatdoost F, Dehghan A, Surkan PJ $\&$ Azadbakht L. Adherence to the DASH and Mediterranean diets is associated with decreased risk for gestational diabetes mellitus. Nutrition 201632 1092-1096. (https://doi.org/10.1016/j. nut.2016.03.006)

8 Assaf-Balut C, de la Torre NG, Durán A, Fuentes M, Bordiú E, Del Valle L, Familiar C, Ortolá A, Jiménez I, Herraiz MA, et al. A Mediterranean diet with additional extra virgin olive oil and pistachios reduces the incidence of gestational diabetes mellitus (GDM): a randomized controlled trial: the St. Carlos GDM prevention study. PLOS ONE 201712 e0185873. (https://doi. org/10.1371/journal.pone.0185873)

9 Schwingshackl L, Schwedhelm C, Galbete C \& Hoffmann G. Adherence to Mediterranean diet and risk of cancer: an updated systematic review and meta-analysis. Nutrients 20179 1063. (https:// doi.org/10.3390/nu9101063)

10 Castejón ML, Rosillo MÁ, Montoya T, González-Benjumea A, Fernández-Bolaños JG \& Alarcón-de-la-Lastra C. Oleuropein downregulated IL-1 $\beta$-induced inflammation and oxidative stress in human synovial fibroblast cell line SW982. Food and Function 20178 1890-1898. (https://doi.org/10.1039/c7fo00210f)
11 Janahmadi Z, Nekooeian AA, Moaref AR \& Emamghoreishi M. Oleuropein attenuates the progression of heart failure in rats by antioxidant and antiinflammatory effects. Naunyn-Schmiedeberg's Archives of Pharmacology 2017390 245-252. (https://doi.org/10.1007/ s00210-016-1323-6)

12 Khalatbary AR \& Zarrinjoei GR. Anti-inflammatory effect of oleuropein in experimental rat spinal cord trauma. Iranian Red Crescent Medical Journal 201214 229-234.

13 Rahiman S, El-Metwally TH, Shrivastava D, Tantry MN \& Tantry BA. Oleuropein and oleic acid: a novel emerging dietary target for human chronic diseases. Indian Journal of Biochemistry and Biophysics 201956 263-268.

14 Pantham P, Aye ILH \& Powell TL. Inflammation in maternal obesity and gestational diabetes mellitus. Placenta 201536 709-715. (https:// doi.org/10.1016/j.placenta.2015.04.006)

15 Al-Azzawie HF \& Alhamdani M-SS. Hypoglycemic and antioxidant effect of oleuropein in alloxan-diabetic rabbits. Life Sciences $2006 \mathbf{7 8}$ 1371-1377. (https://doi.org/10.1016/j.lfs.2005.07.029)

16 Hadrich F, Mahmoudi A, Bouallagui Z, Feki I, Isoda H, Feve B \& Sayadi S. Evaluation of hypocholesterolemic effect of oleuropein in cholesterol-fed rats. Chemico-Biological Interactions 2016252 54-60. (https://doi.org/10.1016/j.cbi.2016.03.026)

17 O'Neill LA \& Hardie DG. Metabolism of inflammation limited by AMPK and pseudo-starvation. Nature 2013493 346-355. (https://doi. org/10.1038/nature11862)

18 Peairs A, Radjavi A, Davis S, Li L, Ahmed A, Giri S \& Reilly CM. Activation of AMPK inhibits inflammation in MRL/lpr mouse mesangial cells. Clinical and Experimental Immunology 2009156 542-551. (https://doi.org/10.1111/j.1365-2249.2009.03924.x)

$19 \mathrm{Wu}$ SB, Wu YT, Wu TP \& Wei YH. Role of AMPK-mediated adaptive responses in human cells with mitochondrial dysfunction to oxidative stress. Biochimica et Biophysica Acta 20141840 1331-1344. (https://doi.org/10.1016/j.bbagen.2013.10.034)

20 O'neill HM. AMPK and exercise: glucose uptake and insulin sensitivity. Diabetes and Metabolism Journal 201337 1-21. (https:// doi.org/10.4093/dmj.2013.37.1.1)

21 Zou C, Zhang Q \& Zhang S. Mogroside IIIE attenuates gestational diabetes mellitus through activating of AMPK signaling pathway in mice. Journal of Pharmacological Sciences 2018138 161-166. (https:// doi.org/10.1016/j.jphs.2018.09.008)

22 Li S, Zhang Y, Sun Y, Zhang G, Bai J, Guo J, Su X, Du H, Cao X, Yang J, et al. Naringenin improves insulin sensitivity in gestational diabetes mellitus mice through AMPK. Nutrition and Diabetes 20199 28. (https://doi.org/10.1038/s41387-019-0095-8)

$23 \mathrm{Lu}$ X, Wu F, Jiang M, Sun X \& Tian G. Curcumin ameliorates gestational diabetes in mice partly through activating AMPK. Pharmaceutical Biology 201957 250-254. (https://doi.org/10.1080/13 880209.2019.1594311)

24 Rigacci S, Miceli C, Nediani C, Berti A, Cascella R, Pantano D, Nardiello P, Luccarini I, Casamenti F \& Stefani M. Oleuropein aglycone induces autophagy via the AMPK/mTOR signalling pathway: a mechanistic insight. Oncotarget $2015635344-35357$. (https://doi.org/10.18632/oncotarget.6119)

25 Mohammed HA, Okail HA, Ibrahim MA \& Emam NM. Influences of olive leaf extract in the kidney of diabetic pregnant mice and their offspring. Journal of Basic and Applied Zoology 201879 1-13.

26 Yao L, Wan J, Li H, Ding J, Wang Y, Wang X \& Li M. Resveratrol relieves gestational diabetes mellitus in mice through activating AMPK. Reproductive Biology and Endocrinology 201513 118. (https:// doi.org/10.1186/s12958-015-0114-0)

27 Caglar GS, Ozdemir ED, Cengiz SD \& Demirtaş S. Sex-hormone-binding globulin early in pregnancy for the prediction of severe gestational diabetes mellitus and related complications. Journal of Obstetrics and Gynaecology Research 201238 1286-1293. (https://doi.org/10.1111/j.1447-0756.2012.01870.x) https://ec.bioscientifica.com https://doi.org/10.1530/EC-20-0466 (c) 2021 The authors Published by Bioscientifica Ltd

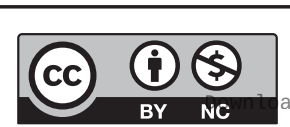

This work is licensed under a Creative Commons Attribution-NonCommercial 4.0 International License. ded from Bioscientifica.com at 04/26/2023 11:21:37AM 
28 Richardson AC \& Carpenter MW. Inflammatory mediators in gestational diabetes mellitus. Obstetrics and Gynecology Clinics of North America 200734 213-224, viii. (https://doi.org/10.1016/j. ogc.2007.04.001)

29 Karacay O, Sepici-Dincel A, Karcaaltincaba D, Sahin D, Yalvaç S, Akyol M, Kandemir O \& Altan N. A quantitative evaluation of total antioxidant status and oxidative stress markers in preeclampsia and gestational diabetic patients in 24-36 weeks of gestation. Diabetes Research and Clinical Practice 201089 231-238. (https://doi. org/10.1016/j.diabres.2010.04.015)

30 Zhang J, Chi H, Xiao H, Tian X, Wang Y, Yun X \& Xu Y. Interleukin 6 (IL-6) and tumor necrosis factor $\alpha$ (TNF- $\alpha$ ) single nucleotide polymorphisms (SNPs), inflammation and metabolism in gestational diabetes mellitus in Inner Mongolia. Medical Science Monitor 201723 4149-4157. (https://doi.org/10.12659/msm.903565)

31 Fujiwara Y, Tsukahara C, Ikeda N, Sone Y, Ishikawa T, Ichi I, Koike T $\&$ Aoki Y. Oleuropein improves insulin resistance in skeletal muscle by promoting the translocation of GLUT4. Journal of Clinical Biochemistry and Nutrition 201761 196-202. (https://doi.org/10.3164/ jcbn.16-120)

32 Wainstein J, Ganz T, Boaz M, Bar Dayan Y, Dolev E, Kerem Z \& Madar Z. Olive leaf extract as a hypoglycemic agent in both human diabetic subjects and in rats. Journal of Medicinal Food 201215 605-610. (https://doi.org/10.1089/jmf.2011.0243)

33 Liu M, Wang J, Huang B, Chen A \& Li X. Oleuropein inhibits the proliferation and invasion of glioma cells via suppression of the AKT signaling pathway. Oncology Reports 201636 2009-2016. (https://doi. org/10.3892/or.2016.4978)

34 Oi-Kano Y, Iwasaki Y, Nakamura T, Watanabe T, Goto T, Kawada T, Watanabe K \& Iwai K. Oleuropein aglycone enhances UCP1 expression in brown adipose tissue in high-fat-diet-induced obese rats by activating $\beta$-adrenergic signaling. Journal of Nutritional Biochemistry 201740 209-218. (https://doi.org/10.1016/j. jnutbio.2016.11.009)

Received in final form 13 October 2020

Accepted 30 November 2020

Accepted Manuscript published online 8 December 2020
This work is licensed under a Creative Commons Attribution-NonCommercial 4.0 International License. ded from Bioscientifica.com at 04/26/2023 11:21:37AM 